\title{
A INFLUÊNCIA DOS SISTEMAS SENSORIAIS NA PLATAFORMA DE FORÇA: ESTUDO DO EQUILÍBRIO CORPORAL EM IDOSAS COM E SEM QUEIXA DE TONTURA
}

\section{Influence of sensorial systems on force plate: study of postural balance in aged women with and without dizziness complaints}

\author{
Clarissa Stefani Teixeira ${ }^{(1)}$, Luiz Fernando Cuozzo Lemos ${ }^{(2)}$, \\ Luis Felipe Dias Lopes ${ }^{(3)}$, Carlos Bolli Mota ${ }^{(4)}$
}

\begin{abstract}
RESUMO
Objetivo: analisar as respostas de dois grupos de idosas (com e sem queixa de tontura) submetidas a diferentes condições sensoriais em uma plataforma de força. Métodos: para a realização desse estudo utilizou-se uma plataforma de força AMTI OR6-5 com uma frequência de aquisição de 100 $\mathrm{Hz}$. Foram realizadas coletas em quatro condições distintas: olhos abertos $(\mathrm{OA})$, olhos fechados (OF) sobre apenas a plataforma de força, olhos abertos (OAA) e olhos fechados (OFA) sobre uma almofada de $20 \mathrm{~cm}$ de espessura. As variáveis analisadas foram o centro de força ântero-posterior (COPap), centro de força médio-lateral (COPml), 95\% da elipse da área, velocidade de oscilação e comprimento da oscilação.Comparações entre os grupos e entre as condições dentro dos grupos, foram realizadas por meio do teste de Duncan. Resultados: os resultados mostraram não haver diferenças entre os grupos em nenhuma variável, porém a condição OFA causou maiores perturbações no equilíbrio corporal das idosas. Conclusão: conclui-se que o aumento da dificuldade imposta pelas quatro condições de testes, alterou a oscilação corporal das idosas avaliadas.
\end{abstract}

DESCRITORES: Equilíbrio Postural; Tontura; Idoso

(1) Graduação em Educação Física; Bolsista Conselho Nacional de Desenvolvimento Científico e Tecnológico; Laboratório de Biomecânica da Universidade Federal de Santa Catarina, UFSC, Florianópolis, SC; Doutoranda em Engenharia da Produção, Universidade Federal de Santa Catarina, Florianópolis, Santa Catarina.

(2) Graduação em Educação Física; Laboratório de Biomecânica da Universidade de Brasília, UnB, Brasília; DF; Mestrando em Educação Física, Universidade de Brasília, Brasília, Distrito Federal.

(3) Graduação em Matemática; Departamento de Estatística da Universidade Federal de Santa Maria, UFSM, Santa Maria, RS; Doutor em Engenharia da Produção, Universidade Federal de Santa Catarina, Florianópolis, Santa Catarina.

(4) Graduação em Engenharia Mecânica; Laboratório de Biomecânica da Universidade Federal de Santa Maria, UFSM, Santa Maria, RS; Doutor em Ciência do Movimento Humano, Universidade Federal de Santa Maria, Santa Maria, Rio Grande do Sul.

Conflito de interesses: inexistente

\section{INTRODUÇÃO}

O controle postural é um aspecto básico para compreender a capacidade que o ser humano tem para exercer suas atividades e manter o corpo em equilíbrio em situações de repouso (equilíbrio estático) e movimento, quando submetido a diversos estímulos (equilíbrio dinâmico) ${ }^{1}$, proporcionando estabilidade e orientação. Segundo Amadio e Serrão ${ }^{2}$ esse controle é baseado na orientação dos arranjos dos segmentos corporais oriundos de informações sensoriais de diferentes fontes.

Para a correta manutenção do equilíbrio corporal, essas informações integradas provêm do sistema vestibular, do proprioceptivo, e do visual, sendo as devidas correções da postura produzidas pelo sistema nervoso central ${ }^{3,4}$. Qualquer falha ou alteração de uma destas vias de informação prejudica 
o recebimento do estimulo pelo sistema nervoso central, alterando a sua resposta. Essa alteração pode causar aos indivíduos desconfortos e impedir ou prejudicar a realização de tarefas do dia-a-dia, assim como levar a ocorrência de quedas quando algum dos sistemas é impedido de recuperar uma perturbação externa ${ }^{5}$.

O sistema vestibular é sensível às acelerações lineares e angulares, enquanto o sistema proprioceptivo é composto por vários receptores que percebem a posição e a velocidade de todos os segmentos corporais, seu contato com objetos externos, inclusive o chão, e a orientação da gravidade ${ }^{6,7}$. As informações visuais baseiam-se nas características externas do ambiente ${ }^{8}$, como por exemplo, a forma, a cor e os movimentos dos objetos e do próprio corpo.

O labirinto pode ser influenciado pelas queixas relatadas pelos indivíduos ${ }^{9,10}$, como, por exemplo, a tontura. Neste caso, a identificação e a busca pela investigação das queixas de tontura é importante, uma vez que, as queixas podem anteceder o acometimento do órgão vestibular ${ }^{8}$. Segundo Kroenke, Hoffman e Einstadter ${ }^{11}$ os problemas de origem vestibular respondem por aproximadamente $50 \%$ dos casos de distúrbio do equilíbrio corporal. Embora alguns indivíduos com sintomas ou problemas vestibulares freqüentemente não apresentem alterações no exame vestibular, estes inferem queixas relacionadas à tontura ${ }^{12}$ e esta por sua vez é considerada como interveniente nas condições de equilíbrio corporal.

As melhoras no equilíbrio corporal são indicadas com a realização de terapias ${ }^{13}$ ou de exercícios físicos ${ }^{8}$ para as queixas de tontura. Porém, estudos que busquem a identificação do tipo de estratégias para manutenção do equilíbrio corporal em indivíduos com e sem queixas de tontura ainda são escassos e necessários.

Diante disso e compreendendo a relevância desse tema, este estudo buscou investigar a influência da manipulação de informações sensoriais sob variáveis do equilíbrio corporal em indivíduos com e sem queixas de tontura. Para a realização do estudo foram formuladas as seguintes hipóteses: hipótese nula $=0$ equilíbrio corporal é igual para os indivíduos com e sem queixas de tontura; hipótese 1 = o equilíbrio corporal é diferente para os indivíduos com e sem queixas de tontura.

\section{MÉTODOS}

Este estudo caracteriza-se, como um estudo descritivo-exploratório, de corte transversal, no qual foi realizado um levantamento de informações ainda pouco investigadas em uma determinada população ${ }^{14}$.

Os indivíduos participantes de grupos de exercícios físicos de uma instituição federal de ensino superior da Região Sul do Brasil, foram convidados a participar do estudo. As atividades realizadas eram hidroginástica com uma frequência de duas vezes na semana. Para o controle do nível de atividade física foi utilizado o Questionário Internacional de Atividades Físicas - IPAQ ${ }^{15}$ e, como recomenda Benedetti, Mazo e Barros, ${ }^{16}$ por se tratar de uma amostra pequena, além da aplicação formal do IPAQ foram realizadas perguntas individuais em forma de entrevista, que possibilitassem visualizar mais amplamente as atividades físicas diárias das idosas.

De um total de 65 indivíduos de ambos os gêneros, foram avaliados aqueles que aceitaram em participar do estudo e que se propuseram a comparecer em todas as avaliações. Para os 60 indivíduos do gênero feminino que aceitaram, foi marcado dia e horário para as coletas a serem realizadas em ambiente de laboratório. Os indivíduos assinaram o Termo de Consentimento Livre e Esclarecido e preencheram uma ficha de dados pessoais, para a obtenção do nome, gênero e idade.

Todos os indivíduos passaram por avaliação otorrinolaringológica. Por meio de uma ficha de anamnese foram excluídos do estudo dois indivíduos com problemas relacionados a aneurisma cerebral, dois com isquemia cerebral transitória, um com problema músculo-esquelético na região dos membros inferiores, e 12 indivíduos que faziam uso de medicamentos associados ao aparelho vestibular.

Para inclusão no estudo e divisão dos grupos (com e sem queixas de tontura), os idosos responderam ao questionário Dizziness Handicap Inventory (DHI) conhecido como DHI Brasileiro adaptado para o português brasileiro por Castro ${ }^{17}$ (2003) e publicado por Castro e colaboradores ${ }^{18}$. Além disso, todos os indivíduos passaram por avaliação otorrinolaringológica, certificando-se da exclusão de problemas vestibulares.

Logo, dos 60 indivíduos do gênero feminino que iniciaram as avaliações foram efetivamente incluídos no estudo apenas 47 indivíduos, sendo $24 \mathrm{com}$ queixas de tontura e 23 sem queixas de tontura. A Tabela 1 ilustra a idade, a estatura e a massa corporal dos idosos de ambos os grupos.

$O$ equilíbrio postural foi coletado por meio de dados cinéticos do centro de força (COP). Para a avaliação cinética foi utilizada uma plataforma de força OR6-5 AMTI (Advanced Mechanical Technologies, Inc.). A frequência de aquisição de dados da plataforma foi de $100 \mathrm{~Hz}$, conforme indicações de 
Tabela 1 - Caracterização dos indivíduos do grupo de estudo

\begin{tabular}{cccc}
\hline Grupos & Idade (anos) & Estatura (m) & Massa corporal $\mathbf{( k g )}$ \\
\hline Com queixas de tontura & $64,43 \pm 6,29$ & $1,54 \pm 0,07$ & $70,67 \pm 9,16$ \\
Sem queixas de tontura & $67,56 \pm 5,28$ & $1,56 \pm 0,06$ & $71,8 \pm 12,70$ \\
\hline
\end{tabular}

Freitas e Duarte ${ }^{19}$. Para a avaliação os indivíduos foram instruídos a posicionar-se em cima da plataforma de força com os pés separados, na largura do quadril, respeitando as características físicas de cada um, com a cabeça direcionada à frente, os olhos focando um ponto fixo a sua frente, a dois metros de distancia e os braços ao longo do corpo. A plataforma foi demarcada na primeira tentativa de cada indivíduo, conforme a base de apoio, e repetida nas demais para assegurar a mesma base em todos os testes, conforme procedimentos expressos na literatura ${ }^{20,21}$. Além disso, as idosas fizeram uso de suas lentes corretivas utilizadas habitualmente.

Foram analisadas quatro diferentes condições de equilíbrio, com os indivíduos descalços, sendo: condição 1: olhos abertos, e idosas com os pés diretamente sobre a plataforma (OA); condição 2: olhos fechados e idosas com os pés diretamente sobre a plataforma (OF); condição 3: olhos abertos e idosas sob uma almofada de $20 \mathrm{~cm}$ de espessura; dimensões de $50 \mathrm{~cm} \times 50 \mathrm{~cm}$ (OAA); e condição 4: olhos fechados e idosas sob a almofada de 20 $\mathrm{cm}$ de espessura; dimensões de $50 \mathrm{~cm} \times 50 \mathrm{~cm}$ (OFA). Foram realizadas três tentativas para cada indivíduo em cada condição, de forma randomizada, por sorteio. Cada condição teve duração de 20 segundos após a estabilização visual do COP, conforme procedimentos de Teixeira e colaboradores ${ }^{21}$. Foi solicitado aos indivíduos que, entre cada condição analisada, descansassem um minuto sentados em uma cadeira colocada próxima as coletas.

Cada condição objetivou a sensibilização de um ou mais sistemas sensoriais. A condição 2 tem a função de retirar a informação do sistema visual através dos olhos fechados, dando ênfase no sistema proprioceptivo e no vestibular. Nas condições 3 e 4 o objetivo foi dessensibilizar o sistema proprioceptivo com o uso da almofada. Nessas condições, ainda é realizada a variação olhos abertos (condição 3) e olhos fechados (condição 4), o que faz com que o corpo necessite de uma maior solicitação do sistema visual e vestibular na condição 3 e do vestibular na condição 4 .

As variáveis analisadas foram a amplitude de deslocamento na direção ântero-posterior (COPap) e na direção médio-lateral (COPml) do centro de força, 95\% da elipse da área (95\% área), velocidade de oscilação (Vel) e comprimento da oscilação (Comp), por meio das seguintes fórmulas:

$$
\begin{gathered}
C O P_{a p}=(M y-h \cdot F x) / F z \\
C O P_{m l}=(M x-h \cdot F y) / F z \\
V m=\frac{L}{n \cdot \Delta t} \\
C=\sum_{i=2}^{n} \sqrt{\left(x_{i}-x_{i-1}\right)^{2}+\left(y_{i}-y_{i-1}\right)^{2}} \\
\text { Área }=(2) \cdot(3,1415963) \cdot(3) \sqrt{\sigma_{x}^{2} \sigma_{y}^{2}-\sigma_{x y}^{2}}
\end{gathered}
$$

Onde:

COPap = coordenada do centro de força na direção ântero-posterior;

COPml = coordenada do centro de força na direção médio-lateral;

$M x=$ momento em torno do eixo ântero-posterior; My = momento em torno do eixo médio-lateral;

$\mathrm{Fx}=$ componente ântero-posterior da força de reação do solo;

Fy = componente médio-lateral da força de reação do solo;

$\mathrm{Fz}=$ componente vertical da força de reação do solo;

$\mathrm{h}$ = distância da superfície até o centro geométrico da plataforma de força;

$\mathrm{Vm}=$ velocidade média;

$\mathrm{L}=$ comprimento total do caminho do COP;

$\mathrm{n}=$ número total de quadros;

$\Delta \mathrm{t}=$ intervalo de tempo;

$\mathrm{C}=$ comprimento total da trajetória;

$\mathrm{x}_{\mathrm{i}}=$ coordenada do eixo $\mathrm{x}$;

$y_{i}=$ coordenada do eixo $y$;

$\sigma^{2}{ }_{x}=$ Variância do $x$;

$\sigma^{2}=$ Variância do $y$;

$\sigma_{x y}^{2^{y}}=$ Covariância de $x$ e $y$. 
As informações provenientes da plataforma de força foram tratadas em ambiente Matlab (Matlab 7.0, The MathWorks, Inc.) para a filtragem dos dados, utilizando-se um filtro Butterworth passa baixas de quinta ordem, com frequência de corte de $10 \mathrm{~Hz}$.

O estudo foi aprovado sob protocolo 0044.0243.000-07 no Comitê de Ética em Pesquisa com seres humanos da Universidade Federal de Santa Maria.

Para análise dos dados foi utilizada a estatística descritiva por meio da média e do desvio padrão. A normalidade dos dados foi verificada por meio do teste Shapiro-Wilk, que mostrou que os dados podem ser considerados como tendo distribuição normal. Para as comparações, a análise estatística se deu em dois momentos, sendo realizada primeiramente uma comparação entre as condições (olhos abertos, fechados, com a almofada e sem a almofada dentro de cada do grupo) para identificar a influência das alterações sensoriais no grupo. No segundo momento, foi realizada comparação entre as variáveis de cada grupo, com o intuito de identificar a diferença dos indivíduos com e sem queixas de tontura. Para essas comparações foi utilizada Anova One-Way. Como algumas condições se mostraram estatisticamente diferentes, foi aplicado o teste de Duncan para identificação dessas diferenças. Para a comparação das variáveis entre os grupos com e sem queixas de tontura, foi utilizado o teste $t$ de Student independente. O nível de significância utilizado para todos os testes foi de 5\%. Para os cálculos estatísticos de todas as análises foi utilizado o programa Statistica® 7.1 for Windows $₫$.

\section{RESULTADOS}

Os resultados obtidos, das variáveis estudadas nos grupos com queixas de tontura, estão ilustrados na Tabela 2. Os resultados da análise estatística, por meio do teste de Duncan, comparando a influência das quatro condições dentro do grupo, estão ilustrados pelas letras ${ }^{a} e^{b}$, em que as médias seguidas de letras diferentes indicam diferenças estatisticamente significantes $(p<0,05)$ entre as variáveis.

A Tabela 3 ilustra os valores da amplitude de deslocamento do centro de força na direção ânteroposterior e médio-lateral, da área da elipse, da velocidade de oscilação e do comprimento da oscilação, nas condições testadas para o grupo sem queixa de tontura. A análise de variância de Duncan indica, por meio das letras ${ }^{a} e^{b}$, diferença estatisticamente significante entre as variáveis estudadas. Estas análises mostram que apenas as variáveis COPap e COPml mostraram-se diferentes no grupo sem queixas de tontura considerando a manipulação das informações sensoriais.

$\mathrm{Na}$ Tabela 4 está ilustrado o nível de probabilidade de significância obtido com as comparações das variáveis COPap, COPml, 95\% área, Vel e Comp, entre os grupos (com e sem queixas de tontura) nas quatro condições testadas (OA, OF, OAA, OFA).

De acordo com os resultados da Tabela 4, não foram encontradas diferenças estatisticamente significantes entre os grupos nas quatro condições avaliadas, o que demonstra estabilidade semeIhante entre os grupos, considerando as condições testadas.

Tabela 2 - Média e desvio padrão das variáveis nas quatro condições no grupo com queixas de tontura e diferenças estatísticas encontradas

\begin{tabular}{ccccccc}
\hline Condições & Variáveis & $\begin{array}{c}\text { COPap } \\
(\mathbf{c m})\end{array}$ & $\begin{array}{c}\text { COPml } \\
(\mathbf{c m})\end{array}$ & $\begin{array}{c}95 \% \text { área } \\
\left(\mathbf{c m}^{2}\right)\end{array}$ & Vel (cm/s) & $\begin{array}{c}\text { Comp } \\
\text { (cm) }\end{array}$ \\
\hline \multirow{2}{*}{ OA } & Média & $2,50^{\mathrm{ab}}$ & $1,85^{\mathrm{a}}$ & $3,76^{\mathrm{ab}}$ & $4,15^{\mathrm{a}}$ & $94,33^{\mathrm{a}}$ \\
& Desvio padrão & 5,72 & 5,34 & 14,26 & 4,31 & 171,80 \\
\multirow{2}{*}{ OF } & Média & $2,02^{\mathrm{b}}$ & $1,10^{\mathrm{a}}$ & $1,80^{\mathrm{b}}$ & $4,42^{\mathrm{a}}$ & $80,03^{\mathrm{a}}$ \\
& Desvio padrão & 1,02 & 0,77 & 2,08 & 2,31 & 53,52 \\
\multirow{2}{*}{ OAA } & Média & $2,50^{\mathrm{ab}}$ & $1,61^{\mathrm{a}}$ & $3,18^{\mathrm{ab}}$ & $3,92^{\mathrm{a}}$ & $71,01^{\mathrm{a}}$ \\
& Desvio padrão & 1,15 & 1,15 & 3,67 & 1,65 & 40,88 \\
\multirow{2}{*}{ OFA } & Média & $4,02^{\mathrm{a}}$ & $2,03^{\mathrm{a}}$ & $6,87^{\mathrm{a}}$ & $5,06^{\mathrm{a}}$ & $92,90^{\mathrm{a}}$ \\
& Desvio padrão & 1,89 & 0,95 & 5,00 & 2,69 & 62,13 \\
\hline
\end{tabular}

ab Médias seguidas de letras diferentes indicam diferenças estatisticamente significantes $(p<0,05)$ entre as condições do estudo para o grupo com queixas de tontura m cada variável analisada 
Tabela 3 - Média e desvio padrão das variáveis nas quatro condições no grupo sem queixas de tontura e diferenças estatísticas encontradas

\begin{tabular}{|c|c|c|c|c|c|c|}
\hline Condições & Variáveis & $\begin{array}{c}\text { COPap } \\
\text { (cm) }\end{array}$ & $\begin{array}{c}\text { COPml } \\
\text { (cm) }\end{array}$ & $\begin{array}{c}95 \% \text { área } \\
\left(\mathrm{cm}^{2}\right)\end{array}$ & $\operatorname{Vel}(\mathrm{cm} / \mathrm{s})$ & $\begin{array}{c}\text { Comp } \\
\text { (cm) }\end{array}$ \\
\hline \multirow{2}{*}{ OA } & Média & $1,41^{b}$ & $0,76^{b}$ & $0,95^{b}$ & $4,33^{a}$ & $79,8^{a}$ \\
\hline & Desvio padrão & 0,58 & 0,35 & 0,56 & 4,08 & 86,16 \\
\hline \multirow{2}{*}{ OF } & Média & $2,11^{b}$ & $1,09^{b}$ & $1,73^{b}$ & $4,46^{a}$ & $83,44^{\mathrm{a}}$ \\
\hline & Desvio padrão & 0,88 & 0,56 & 1,35 & 2,85 & 61,96 \\
\hline \multirow{2}{*}{ OAA } & Média & $2,55^{\mathrm{b}}$ & $1,57^{b}$ & $3,41^{\mathrm{ab}}$ & $4,81^{a}$ & $83,26^{a}$ \\
\hline & Desvio padrão & 1,16 & 1,03 & 4,48 & 2,63 & 49,07 \\
\hline \multirow{2}{*}{ OFA } & Média & $4,95^{a}$ & $3,20^{a}$ & $21,42^{a}$ & $6,01^{a}$ & $110,33^{\mathrm{a}}$ \\
\hline & Desvio padrão & 3,65 & 3,86 & 61,87 & 2,69 & 60,80 \\
\hline
\end{tabular}

ab Média seguida de letras diferentes indicam diferenças estatisticamente significantes $(p<0,05)$ entre as condições do estudo para 0 grupo sem queixas de tontura em cada variável analisada

Tabela 4 - Valor do nível de probabilidade de significância entre os grupos com e sem queixas de tontura para as variáveis em relação às condições

\begin{tabular}{ccccc}
\hline \multirow{2}{*}{ Variáveis analisadas } & \multicolumn{4}{c}{ Condições } \\
\cline { 2 - 5 } & OA & OF & OAA & OFA \\
\hline COPap $(\mathrm{cm})$ & 0,37 & 0,76 & 0,89 & 0,27 \\
COPml $(\mathrm{cm})$ & 0,33 & 0,95 & 0,90 & 0,16 \\
$95 \%$ área $\left(\mathrm{cm}^{2}\right)$ & 0,35 & 0,88 & 0,85 & 0,26 \\
Vel $(\mathrm{cm} / \mathrm{s})$ & 0,89 & 0,95 & 0,17 & 0,23 \\
Comp $(\mathrm{cm})$ & 0,72 & 0,84 & 0,36 & 0,34 \\
\hline
\end{tabular}

\section{DISCUSSÃO}

A tontura pode acometer todas as faixas etárias. O maior destaque entre os estudos é dado principalmente aos indivíduos que estão envelhecendo, uma vez que, há consequência de quedas em função das queixas de tontura ${ }^{22}$. Mesmo que existam relações entre as queixas de tontura e a instabilidade corporal, o presente estudo não identificou diferenças estatisticamente significantes relacionadas à estabilidade mesmo com a manipulação dos sistemas sensoriais.

A sensibilização dos sistemas sensoriais e suas relações com a manutenção da postura em pé, foi foco de análise ${ }^{23}$ utilizando um torniquete acima da articulação do tornozelo, visando diminuir a corrente sanguínea em jovens adultos saudáveis. A diminuição no fluxo causou decréscimo da atuação do sistema proprioceptivo na manutenção do equilíbrio corporal, mostrando associação entre o grau das perdas sensoriais e os aumentos de oscilações corporais. Visando uma manipulação análoga do sistema proprioceptivo, utilizou-se, no presente estudo, a almofada para causar o conflito sensorial.
A influência proprioceptiva causou maiores oscilações nos indivíduos avaliados, na maioria das condições estudadas ${ }^{23}$. No grupo com queixas de tontura, observou-se diferenças estatisticamente significantes nas variáveis COPap e na elipse dos $95 \%$ da área, entre a condição de OF e OFA (Tabela 2), o que demonstra que a ausência do estímulo visual somada com o conflito proprioceptivo (condição OFA) causa desequilíbrios posturais. Essa situação faz com que haja uma maior solicitação do sistema vestibular ${ }^{8}$. Além disso, o sistema vestibular, quando afetado, atua como o principal responsável pelas vertigens e tonturas que causam os desequilíbrios corporais ${ }^{24}$.

Com relação ao grupo sem as queixas de tontura, as diferenças entre os três sistemas ocorreram de forma similar ao grupo de indivíduos com queixas. Porém, as diferenças incidiram na condição OFA quando comparada com as demais condições, nas variáveis COPap e COPml (Tabela 3). Assim, é possível inferir que indivíduos sem queixas de tontura sofrem um grande desafio em manter a estabilidade nessas condições, com manipulações das entradas sensoriais. Um exemplo de estudo 
que buscou avaliar indivíduos sem queixas, permitiu identificar que com os olhos fechados há uma maior oscilação corporal, mesmo considerando indivíduos sem problemas de saúde 4 . Além disso, comparando os resultados obtidos pelos mesmos autores ${ }^{4}$ com os do presente estudo, nota-se que os ginastas avaliados apresentaram valores menores (ou seja, melhor equilíbrio corporal) que os indivíduos aqui avaliados para a velocidade média de oscilação nas condições OA, OF e OAA. Porém, na condição OFA houve similaridade nos valores, mostrando menor tempo em equilíbrio quase-estático nos indivíduos do presente estudo, quando comparados com as ginastas.

Com base nesses dados, visualiza-se que os grupos avaliados tiveram menor velocidade na ação neuromuscular em resposta a oscilação corporal do seu centro de gravidade, causando assim, o aumento do valor de seu COP (maiores oscilações corporais), o que pode ser atribuído à idade mais elevada dos indivíduos do presente estudo, quando comparados às ginastas 4 .

Alguns pesquisadores ${ }^{25}$ realizaram testes de manipulações sensoriais, similares aos do presente estudo, em indivíduos com diferentes níveis de acuidade visual com o objetivo de induzir a utilização de estratégias, o que causou um aumento dos valores da oscilação dos indivíduos estudados, para assim, evitar possíveis quedas. No presente estudo, possivelmente novas e variadas estratégias ocorreram em função das condições impostas aos indivíduos, pois os valores visualizados no grupo sem tontura, na condição OFA, foram maiores que nas condições OA e OF, para a variável área (Tabela 3).

Outro fato importante ao qual se ressalta, é que existem perdas naturais no ser humano com o avançar da idade, relacionadas às características físicas, e assim ocorrem maiores dificuldades para manutenção da postura em equilíbrio estático ${ }^{26,27}$. Porém, segundo Vrieling e colaboradores $^{28}$ os indivíduos são hábeis para o controle do equilíbrio corporal, sendo capazes de compensar as deficiências provenientes de alguns dos sistemas para o ajustamento ao meio ambiente em condições necessárias.

Especificamente sobre as adaptações visuais, O'Connor e colaboradores ${ }^{29}$ indicaram que indivíduos conseguem habituar as perturbações de acordo com o número de exposições. Estas indicações mostram que um dos impactos, em indivíduos com idades mais avançadas, seria relacionado à capacidade de modificar rapidamente os estímulos sensoriais para a estabilização postural.

Os grupos avaliados pelo estudo, de acordo com as comparações realizadas, podem ser considerados como homogêneos para as variáveis de equilíbrio, visto que não foram identificadas diferenças estatisticamente significante com as comparações nas diferentes condições sensoriais (Tabela 4). Estas indicações podem estar relacionadas pelas afirmações que tratam da tontura como sendo um mal transitório, ou seja, indivíduos afligidos por ela no período agudo têm muitas dificuldades de deslocamentos ou mesmo para manter-se em pé. Em contrapartida, quando os indivíduos não estão em crise, há possibilidade de realização das tarefas da vida diária de forma quase que normalmente ${ }^{30}$.

Diferentemente dos resultados do presente estudo, o estudo de Bastos e colaboradores ${ }^{31}$ buscaram analisar o equilíbrio corporal de adultos com queixas de tonturas e realizaram comparações com um grupo controle sem queixas. As variáveis analisadas foram a amplitude média do deslocamento do centro de força nos sentidos médio-lateral e ântero-posterior, a velocidade de deslocamento do centro de força nos sentidos médio-lateral e ântero-posterior e a área da elipse. Os resultados das variáveis favoreceram o grupo controle que mostrou valores estatisticamente melhores, para o equilíbrio corporal, em comparação aos indivíduos acometidos pelas queixas.

O comprimento de oscilação não se mostrou sensível nem aos grupos nem às condições, o que difere do estudo de Haibach e colaboradores ${ }^{32}$. Esses autores analisaram o equilíbrio manipulando a informação visual com a utilização de movimentos virtuais. Foram avaliados três grupos diferentes (adultos sadios, idosos sem queixa de quedas e idosos com queixa de quedas) em cinco diferentes amplitudes de imagens virtuais. Os resultados mostraram que o comprimento da oscilação dos adultos foi o menor, seguido pelos idosos sem queixas de quedas e por último, os idosos com queixas de quedas. Mesmo que os grupos de indivíduos do presente estudo não tenham apresentado diferenças entre si, pode-se dizer que os valores são intermediários aos idosos sem e com queixas de quedas do estudo de Haibach e colaboradores $^{32}$, o que indica para os participantes do presente estudo, maior desequilíbrio para o grupo sem queixa de tontura e maior equilíbrio para o grupo com queixas de tontura quando comparados aos resultados do trabalho de Haibach e colaboradores ${ }^{32}$. Tais achados podem ser explicados pelo fato de que a queixa de tontura não representa a queda, mas sim uma predisposição para o fenômeno. Portanto, infere-se que as pessoas com queixa de quedas apresentam valores de comprimento de oscilação do COP maiores que pessoas com queixas de tonturas. 


\section{CONCLUSÃO}

Com o aumento da dificuldade imposta pelas quatro diferentes condições, expressas neste estudo, pode-se vislumbrar que diante da sensibilização da informação proprioceptiva e com a ausência da visual, os indivíduos apresentaram maiores oscilações. Porém, a manutenção da postura em pé foi mantida mesmo sofrendo vários desafios.
Com relação a comparação entre os grupos com e sem queixas de tontura aceita-se a hipótese nula, uma vez que não foram encontradas diferenças estatisticamente significante no equilíbrio corporal em diferentes condições sensoriais.

A realização de novos estudos para identificação das instabilidades com relação à crise de tontura é importante para o desenvolvimento e o direcionamento do tipo de terapia a ser realizada pelos indivíduos com queixa de desequilíbrio postural.

\begin{abstract}
Purpose: to analyze the responses of two groups of aged women (with and without dizziness complaints) under different sensorial conditions on a force platform. Methods: to analyze the data used is a force platform AMTI OR6-6 under a frequency of $100 \mathrm{~Hz}$. Samples were collected in four different conditions, which are, as it follows: open eyes (OE), closed eyes (CE) only on the force platform, open eyes (OE) and closed eyes (CE) on a cushion of $20 \mathrm{~cm}$ thickness. The examined variables were the anterior-posterior center of power (COPap), center of medium-strength side (COPml), $95 \%$ of the ellipse area, length and speed of oscillation. Comparisons were made between the group and between conditions within groups, through the test of Duncan. Results: results showed no differences among the groups in any variable, but the CE condition caused the greatest disruption as for the elderly women. Conclusion: we may conclude that the increase in the difficulty imposed by the four assessed conditions, changed the body oscillation of the evaluated elderly women.
\end{abstract}

KEYWORDS: Postural Balance ; Dizziness; Aged

\section{REFERÊNCIAS}

1. López JR, Fernández NP. Caracterización de la interacción sensorial en posturografía. Acta Otorrinolaringol Esp. 2004; 55:62-6.

2. Amadio AC, Serrão JC. Contextualização da biomecânica para a investigação do movimento: fundamentos, métodos e aplicações para análise da técnica esportiva. Rev Bras Educ Fís Esp. 2007; 21:61-85.

3. Bonfim TR, Barela JA. Efeito da manipulação da informação sensorial na propriocepção e no controle postural. Fisio em Mov. 2007; 20:107-17.

4. Harringe ML, Halvorsen K, Renström P, Werner S. Postural control measured as the center of pressure excursion in young female gymnasts with low back pain or lower extremity injury. Gait Posture. 2008; 28:38-45.

5. Liu W, Kim SH, Long JT, Pohl PS, Duncan PW. Anticipatory postural adjustments and the latency of compensatory stepping reactions in humans. Neuroci Lett. 2003; 336:1-4.
6. Duarte M. Análise estabilográfica da postura ereta humana quasi-estática. São Paulo. [livre-docência]. São Paulo (SP): Universidade de São Paulo; 2000. 7. Winter D. Human balance and posture during standing and walking. Gait Posture. 1995; 3:193-214. 8. Teixeira CS. Hidroginástica na reabilitação vestibular de idosos com queixas de tontura. [dissertação]. Santa Maria (RS): Universidade Federal de Santa Maria; 2008.

9. Gushikem P, Caovilla HH, Ganança MM. Avaliação otoneurológica em idosos com tontura. Acta ORL [periódico na Internet] 2003; 21: [8 p]. Disponível em: http://www.actaorl.com.br/detalhe artigo.asp?id=3. Acesso em 03 abr 2009.

10. Ruwer SL, Rossi AG, Simon LF. Equilíbrio no idoso. Rev Bras Otorrinolaringol. 2005; 71:298-303. 11. Kroenke K, Hoffman RM, Einstadter D. How common are various causes of dizziness? A critical review. South Med J. 2000; 93(2):160-7.

12. Tiensoli LO, Couto ER, Mitre El. Fatores associados à vertigem ou tontura em Indivíduos com exame vestibular normal. Rev CEFAC. 2004; 6(1):94-100. 
13. Resende SM, Rassi CM, Viana FP. Efeitos da hidroterapia na recuperação do equilíbrio e prevenção de quedas em idosas. Rev Bras Fisioter. 2008; 12(1):57-63.

14. Thomas JR, Nelson JK. Métodos de pesquisa em atividade física. Porto Alegre: ArtMed, 2002.

15. Pardini R, Matsudo $S$, Matsudo $V$, Araújo $\mathrm{T}$, Andrade $\mathrm{E}$, Braggion $\mathrm{G}$, et al. Validation of International Physical Questionnaire (IPAQ): pilot study in Brazilian young adults. Med Sci Sports Exerc. 1997; 29(6):S5-S9.

16. Benedetti TRB, Mazo GZ, Barros MV. Aplicação do Questionário Internacional de Atividade Física para avaliação do nível de atividades físicas de mulheres idosas: validade concorrente e reprodutibilidade teste/reteste. Rev Bras Ciên Mov. 2004; 12(1):25-33.

17. Castro ASO. Dizziness Handicap Inventory: adaptação cultural para o português brasileiro, reprodutibilidade e comparação com os resultados à vestibulometria. [dissertação]. São Paulo (SP): Universidade Bandeirante de São Paulo; 2003.

18. Castro ASO, Gazzola JM, Natour J, Ganança FF. Brazilian version of the Dizziness Handicap Inventory. Pró-Fono. 2007; 19(1):97-104.

19. Freitas SMSF, Duarte M. Métodos de análise do controle postural. Capitulo 1. 2005: [21 p]. [acesso em 2009 fev 11]. Disponível em: http://lob. incubadora.fapesp.br/ portal/p/nec05.pdf

20. Mann L, Kleinpaula JF, Teixeira CS, Rossi AG, Lopes LFD, Mota CB. Investigação do equilíbrio corporal em idosos. Rev Bras Geriatr Gerontol. 2008; 11(2):155-65.

21. Teixeira CS, Lemos LFC, Lopes LFD, Rossi AG, Mota CB. Equilíbrio corporal e exercícios físicos: uma investigação com mulheres idosas praticantes de diferentes modalidades. Acta Fisiatr. 2008; 15(3):154-7.

22. Morettin M, Mariotto LD, Costa Filho OA. Avaliação da efetividade da reabilitação vestibular em pacientes com queixas vestibulares. Arq Int Otorrinolaringol. 2007; 11(3):284-92.

23. Wang TY, Lin SI. Sensitivity of plantar cutaneous sensation and postural stability. Clinical Biomechanics. 2008; 23:493-9.

24. Soares EV. Reabilitação vestibular em idosos com desequilíbrios para marcha. Perspect. 2006; 6:88-100.

25. Ray CT, Horvat M, Croce R, Mason RC, Wolf SL. The impact of vision loss on postural stability and balance strategies in individuals with profound vision loss. Gait Posture. 2008; 28:58-61.

26. Monteiro W, Monteiro FFS, Oliveira AV, Jesus AP, Bueno CS, Oliveira CS. Análise do equilíbrio dinâmico em idosas praticantes de dança de salão. Fisio Mov. 2007; 20:125-36.

27. Teixeira CS, PereiraÉF, Rossi AG. Hidroginástica como meio para manutenção da qualidade de vida e saúde do idoso. Acta Fisiatr. 2007; 14:226-32.

28. Vrieling $A H$, Van Keeken HG, Schoppen $T$, Otten E, Hof AL, Halbertsma JPK, et al. Balance control on a moving platform in unilateral lower limb amputees. Gait Posture. 2008; 28:222-8.

29. O'Connora KW, Loughlina PJ, Redferna MS, Sparto PJ. Postural adaptations to repeated optic flow stimulation in older adults. Gait Posture. 2008; 28:385-91.

30. Handa PR, Kubn AMB, Cunha F, Schaffleln R, Grança FF. Qualidade de vida em pacientes com vertigem posicional paroxística benigna e/ ou doença de Ménière. Rev Bras Otorrinolaringol. 2005; 71(6):776-83.

31. Bastos AGD, Lima MAMT, Oliveira LF. Avaliação de pacientes com queixa de tontura e eletronistagmografia normal por meio da estabilometria. Rev Bras Otorrinolaringol. 2005; 71:305-10.

32. Haibach PS, Slobounov SM, Newell KM. The potencial application of a virtual moving environment for assessing falls in elderly adults. Gait Posture. 2008; 27:303-8.

DOI: 10.1590/S1516-18462010005000110

RECEBIDO EM: 23/06/2009

ACEITO EM: 23/06/2010

Endereço para correspondência:

Clarissa Stefani Teixeira

Centro de Educação Física e Desportos, Labiomec

Faixa de Camobi, km 9

Santa Maria - RS

CEP: 97105-900

E-mail: clastefani@gmail.com 